\title{
Accuracy and clinical outcomes of coronary CT angiography for patients with suspected coronary artery disease: a single-centre study in Singapore
}

\author{
Awesh Shamrao Gambre ${ }^{1}$, MBBS, FRCR, Charlene Liew ${ }^{1}$, MBBS, FRCR, Gayan Hettiarachchi ${ }^{1}$, MD, MBBS,
} Sheldon Shao Guang $\underline{\text { Lee }}^{2}$, MBBS, MRCP, Michael MacDonald ${ }^{2}$, MD, MRCP, Carmen Jia Wen $\underline{K a m}^{3}$, Angeline Choo Choo $\underline{\mathrm{Poh}}^{1}$, MBBS, FRCR

INTRODUCTION This study aimed to assess the accuracy and outcomes of coronary computed tomography angiography (CCTA) performed in a regional hospital in Singapore.

METHODS The Changi General Hospital CCTA database was retrospectively analysed over a 24-month period. Electronic hospital records, catheter coronary angiography (CCA) and CCTA electronic databases were used to gather data on major adverse cardiovascular events (MACE) and CCA results. CCTA findings were deemed positive if coronary artery stenosis $\geq 50 \%$ was reported or if the stenosis was classified as moderate or severe. CCA findings were considered positive if coronary artery stenosis $\geq 50 \%$ was reported.

RESULTS The database query returned 679 patients who had undergone CCTA for the evaluation of suspected coronary artery disease. Of the 101 patients in the per-patient accuracy analysis group, there were six true negatives, one false negative, 81 true positives and 13 false positives, resulting in a negative predictive value of $85.7 \%$ and positive predictive value of $86.2 \%$. The mean age of the study sample was $53 \pm 13$ years and 255 (37.6\%) patients were female. Mean duration of patient follow-up was 360 days. Of the 513 negative CCTA patients, none developed MACE during the follow-up period, and of the 164 positive CCTA patients, 19 (11.6\%) developed MACE $(p<0.001)$.

CONCLUSION Analysis of CCTA studies suggested accuracy and outcomes that were consistent with published clinical data. There was a one-year MACE-free warranty period following negative CCTA findings.

Keywords: coronary angiography, coronary artery disease, coronary stenosis, CT angiography, prognosis

\section{INTRODUCTION}

Coronary artery disease (CAD) is one of the leading causes of mortality and morbidity in Singapore. Catheter coronary angiography (CCA) is considered the gold standard for diagnosing $\mathrm{CAD}$, as it is highly reliable and has the ability to determine the extent, location and severity of coronary obstructive lesions. ${ }^{(1-8)}$ However, CCA is not suitable for all patients with suspected CAD due to its invasive nature and risk of complications. Furthermore, not all conventional angiography procedures proceed to intervention. Weighing the risks of CCA for a patient who may not require intervention may tip the scales in favour of a noninvasive test for diagnosing coronary stenosis. ${ }^{(9,10)}$

The continuous advances in coronary computed tomography angiography (CCTA) technology have also improved the diagnostic performance of noninvasive coronary imaging, and the high negative predictive values (NPVs) afforded by CCTA enable reliable exclusion of significant coronary stenosis. ${ }^{(11,12)}$ Other additional advantages of CCTA include its ability to provide prognostic information and guide management in patients with suspected CAD. ${ }^{(13-16)}$ An accurate, noninvasive alternative evaluation method for diagnosing CAD is highly desirable, particularly in patients with a low-to-intermediate pre-test likelihood of CAD, in whom CAD cannot be diagnosed or excluded based on clinical assessment alone. ${ }^{(10,17,18)}$

\section{METHODS}

The CCTA database at Changi General Hospital (CGH), Singapore, was analysed over a 24-month period from January 2013 to December 2014. Electronic medical records, CCA and CCTA images and reports from the cardiology and radiology Picture Archival and Communication databases were analysed to determine major adverse cardiovascular events (MACE) and CCA results in the patient subsets described in detail below.

CCTA findings were deemed positive if coronary artery stenosis $\geq 50 \%$ was reported or if it was graded as 'moderate' or 'severe', and negative if there was $<50 \%$ stenosis in all coronary arteries. CCA findings were deemed positive if coronary artery stenosis $\geq 50 \%$ was reported. The definition of MACE included the following events: death, acute myocardial infarction, unstable angina, acute coronary syndrome and cardiac revascularisation. Information on MACE was determined from our hospital's electronic medical records.

The database query returned 728 patients who had CCTA performed for the assessment of suspected CAD during the study period. The study population consisted of low-to-intermediate risk patients with an age range of 15-87 years who presented with the chief complaint of acute-onset chest pain and were referred for CCTA by cardiologists, emergency department physicians and other clinicians, at the referring clinician's discretion. A total of 35 patients

${ }^{1}$ Department of Radiology, ${ }^{2}$ Department of Cardiology, ${ }^{3}$ Clinical Trials and Research Unit, Changi General Hospital, Singapore

Correspondence: Dr Awesh Shamrao Gambre, Radiology Resident, Department of Radiology, Changi General Hospital, 2 Simei Street 3 , Singapore 529889. aweshgambre@gmail.com 


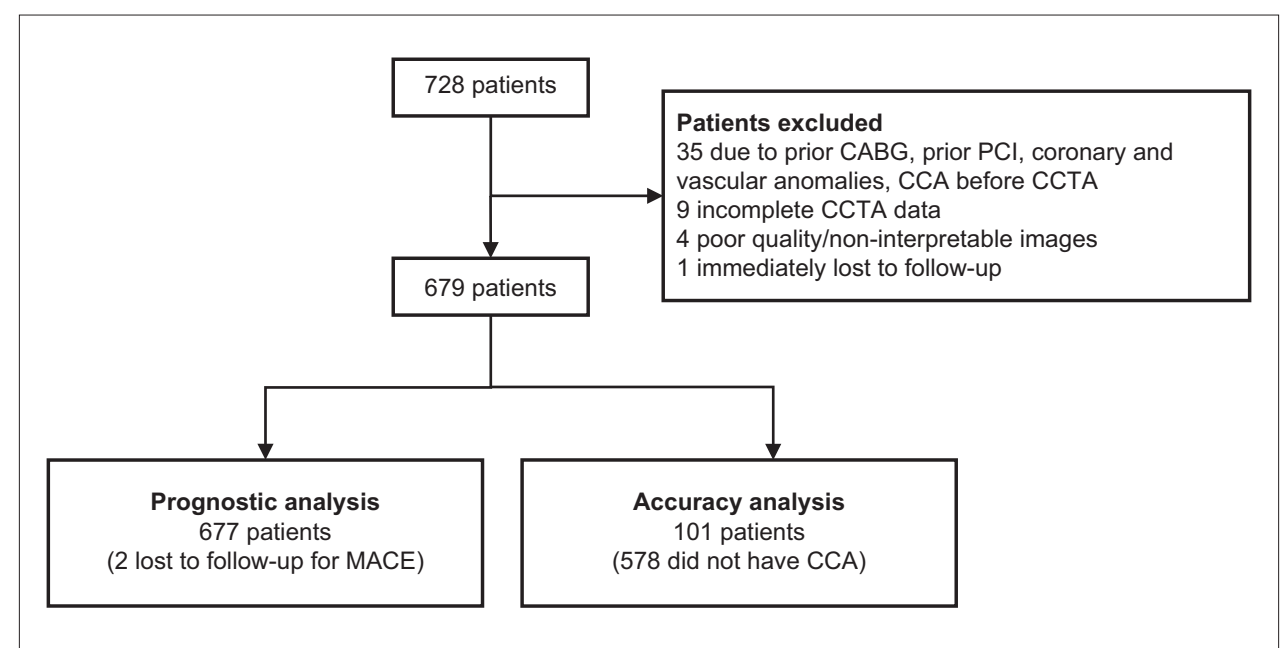

Fig. 1 Flowchart shows the inclusion criteria for the study. CABG: coronary artery bypass graft; CCA: catheter coronary angiography; CCTA: coronary computed tomography angiography; MACE: major adverse cardiovascular events; $\mathrm{PCl}$ : percutaneous coronary intervention

with prior coronary artery bypass graft (CABG) surgeries, anomalous arteries or other vascular anomalies, and those with CCA before CCTA was performed, were excluded from analysis. Another nine patients were excluded due to incomplete CCTA data, four due to non-interpretable images and one was lost to follow-up (Fig. 1).

Patients were analysed in two groups: one group for accuracy analysis and the other for prognostic analysis. Of the 679 patients, 101 (14.9\%) had undergone both CCTA and CCA and were identified for accuracy analysis. 677 patients were evaluated for MACE and used for prognostic analysis. All patients analysed had undergone retrospective or prospective electrocardiography (ECG)-gated CCTA using a 320-row detector scanner (Toshiba Aquilion ONE, Toshiba Medical Systems Corporation, Tochigi, Japan). Patient preparation involved a four-hour fast and insertion of an $18 \mathrm{G}$ cannula in the right antecubital vein. A beta-blocker (metoprolol tartrate; Betaloc, AstraZeneca, Cambridge, UK) was administered intravenously for patients without contraindications and with heart rates $>60$ beats/minute, and $0.5 \mathrm{mg}$ sublingual glyceryl trinitrate was given before the start of the scan. Patients were positioned feet first with arms above their head and asked to perform breath-hold exercises in the scanner to ensure that their heart rate did not fluctuate more than 10\% during the breath hold.

Initial anteroposterior scout computed tomography was performed, centred at the sternal notch with coverage from the supraclavicular region to lung bases. Scan coverage from the pulmonary trunk to the apex of the heart included the entire heart with a 2-3 cm allowance for anatomical excursion during breath hold. $60 \mathrm{~mL}$ iodinated contrast and $40 \mathrm{~mL}$ saline bolus chaser was injected intravenously via a dual-pump injector at a rate of $4.5 \mathrm{~mL} /$ second as part of the acquisition protocol. A bolus-tracking method was used to trigger the scan acquisition (monitoring level: carina; monitoring delay: five seconds; placement of region of interest: descending aorta, Hounsfield unit threshold: 180). ECG dose modulation was used for radiation dose reduction. The scan acquisition was done using the parameters in Table I.

Categorical data was presented as frequency (percentage). Continuous data was presented as mean \pm standard deviation for parametric distribution and median (interquartile range) for
Table I. Multidetector coronary CTA scan acquisition parameters.

\begin{tabular}{|c|c|}
\hline Parameter & Value \\
\hline$k V p$ & 120/100 (based on patient size) \\
\hline $\mathrm{mA}$ & Sure exposure \\
\hline Slice width/index & $0.5 / 0.25 \mathrm{~mm}$ \\
\hline Volume width/index & $0.5 / 0.25 \mathrm{~mm}$ \\
\hline Collimation & $0.5 \mathrm{~mm}$ (volume scan) \\
\hline Kernel & FC 05 (cardiac CTA sharp volume) \\
\hline Helical pitch & Nil (volume scan) \\
\hline Rotation & $0.35 \mathrm{~s}$ \\
\hline \multirow[t]{6}{*}{ Image to PACS } & $\begin{array}{l}\text { Noncontrast axial, calcium score } \\
\text { images and report (if calcium } \\
\text { score was done) }\end{array}$ \\
\hline & - Lung window $3 / 3 \mathrm{~mm}$ \\
\hline & - $0.5 \mathrm{~mm}$ volume for best series \\
\hline & $\begin{array}{l}2 \text { curved MIPs for each artery } \\
\text { (LAD, LCX, RCA) }\end{array}$ \\
\hline & $\begin{array}{l}\text { Three-dimensional VR images } \\
\text { of (1) LM, (2) LM bifurcation, (3) } \\
\text { LAD, (4) LCX, (5) RCA, (6) PDA + } \\
\text { PLV, (7) reverse spider view }\end{array}$ \\
\hline & $\begin{array}{l}\text { All images generated by } \\
\text { radiologist }\end{array}$ \\
\hline Image to archive & Volume $0.5 / 0.25 \mathrm{~mm}$ for all phases \\
\hline
\end{tabular}

CTA: computed tomography angiography; LAD: left anterior descending artery; LCX: left circumflex artery; LM: left main artery; MIP: maximum intensity projection; PACS: Picture Archival and Communication database; PDA: posterior descending artery; PLV: posterior left ventricular artery; RCA: right coronary artery; VR: volume rendering

non-parametric distribution. In prognostic analysis, the association between CCTA and MACE was assessed by chi-square test. A twotailed $p$-value $<0.05$ was considered statistically significant. In accuracy analysis, the accuracy, sensitivity, positive predictive value (PPV), specificity and NPV of CCTA were evaluated for correct identification of the outcome of CCA. A measure of at least $70 \%$ was considered as clinically acceptable. Prevalence of CCA was also reported. Statistical data analysis was performed with IBM SPSS Statistics version 19.0 (IBM Corp, Armonk, NY, USA). 


\section{RESULTS}

In the per-patient accuracy analysis group comprising 101 patients, there were six true negatives, one false negative, 81 true positives and 13 false positives, resulting in a prevalence of $81.2 \%$, accuracy of $86.1 \%$, sensitivity of $98.8 \%$, PPV of $86.2 \%$, specificity of $31.6 \%$ and NPV of $85.7 \%$. However, specificity and NPV could not be accurately calculated since the vast majority of negative CCTA patients did not have subsequent CCA.

In the per-vessel analysis group, CCTA was compared with CCA for the left main artery, left anterior descending artery (LAD), left circumflex artery (LCX) and right coronary artery (RCA) individually. There were 15 false-negative vessels - two in the $L A D$, five in the RCA and eight in the LCX. Further evaluation of CCTA images revealed that in the false-negative group, one LAD, three RCA and one LCX lesions were obscured by heavily calcified plaques and reported as $<50 \%$ stenosed. Two LCX positive lesions were missed due to vein crossover and another due to the poor quality of the images obscuring the LCX origin where the lesion was located. One RCA lesion was missed due to poor image quality and another RCA lesion was missed due to diffuse plaque that made it difficult to appreciate the amount of narrowing. Among the false positives, seven out of 13 studies had been performed on patients who had high calcium scores in the range of 499-4,650 units using the Agatston scoring method, which is known to have an adverse effect on luminal assessment during CCTA due to a phenomenon known as 'blooming' artefact (Fig. 2).

The mean age of the study sample was $53 \pm 13$ (range $15-87)$ years and 255 (37.6\%) patients were female. The mean duration of patient follow-up was 360 (range 120-840) days. Of 513 negative CCTA patients, none went on to develop MACE during the follow-up period, and of the 164 positive CCTA patients, $19(11.6 \%)$ developed MACE $(p<0.001)$. Among the patients who developed MACE, three patients were admitted with unstable angina or acute coronary syndrome and a total of $15(9.1 \%)$ revascularisation procedures were subsequently performed. The angiograms of a patient with positive CCTA who developed MACE are shown in Fig. 3. The 55-year-old Chinese man presented with chest pain without ECG changes or a rising trend of cardiac enzymes. He underwent conventional CCA that confirmed the presence of severe stenosis in the mid $\mathrm{LAD}$, which was revascularised by balloon angioplasty.

\section{DISCUSSION}

The main objective of the study was to assess whether the accuracy of $\mathrm{CGH}^{\prime}$ s CCTA service is comparable with previously published single-centre trial data, and if CCTA is a useful tool for excluding patients who go on to develop MACE. The study was designed to mirror the real-world situation, especially in the context of a busy clinical service and emergency department that relies on CCTA to triage patients for discharge or admission, and as a decision-making tool for assessing the need for further investigation with CCA. ${ }^{(17,19-21)}$ A comparison showed that our results are commensurate with the available literature (Table II), with a good NPV of $85.7 \%$ and a very high sensitivity of $99.0 \%$. The overall accuracy of CCTA compared to CCA was $86.1 \%$.

The present study also sought to determine whether a negative CCTA prognostically conferred a low risk for MACE during follow-up, and this was found to be true: CCTA had an excellent NPV of $100 \%$ for cases that went on to develop MACE within 12 months. This implied a calculated average one-year MACE-free warranty period for negative CCTA. The $0 \%$ rate of MACE at a mean of 12 months' follow-up is comparable with the published data for negative myocardial perfusion scans and stress echocardiography. Metz et $\mathrm{al}^{(18)}$ found that the annual event rates for a negative myocardial perfusion scan and stress echocardiogram were $0.45 \%$ and $0.54 \%$ per year, respectively. Studies by Genders et $\mathrm{al}^{(21)}$ and Min et al ${ }^{(22)}$ have suggested that CCTA is an excellent cost-effective triage test prior to CCA in patients with low-to-intermediate pre-test probability of CAD. Suh et $\mathrm{al}^{(23)}$ also demonstrated the usefulness of CCTA for selecting CABG surgery candidates in patients suspected of CAD. In addition, it is an excellent tool for assessment of both grafts
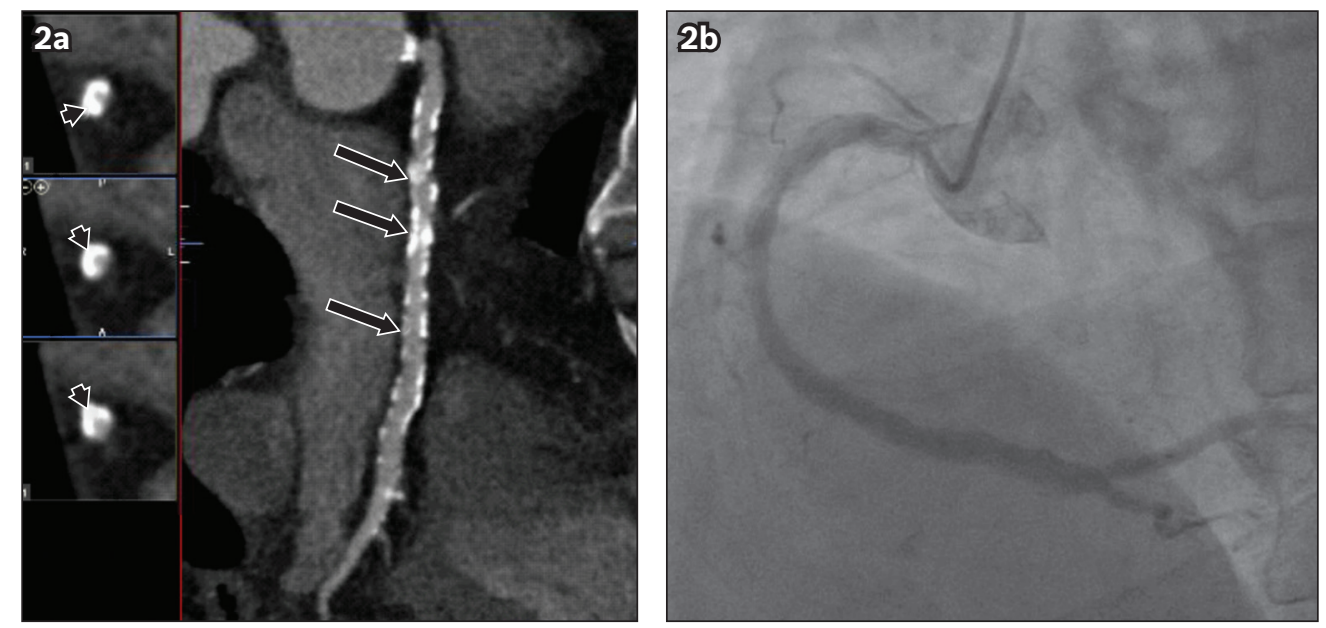

Fig. 2 (a) Contrast-enhanced coronary computed tomography angiography (CCTA) curved planar reformation image of the right coronary artery shows diffuse calcified plaque throughout the vessel (arrows). The calcium score was 4,650 units, reflecting the large volume of calcified plaque, most of it causing marked 'blooming' artefact (arrowheads), which made luminal assessment difficult. (b) Subsequent catheter coronary angiogram shows the relative lack of significant luminal stenosis, highlighting the difficulties posed by heavily calcified plaque resulting in false-positive CCTA reports. 

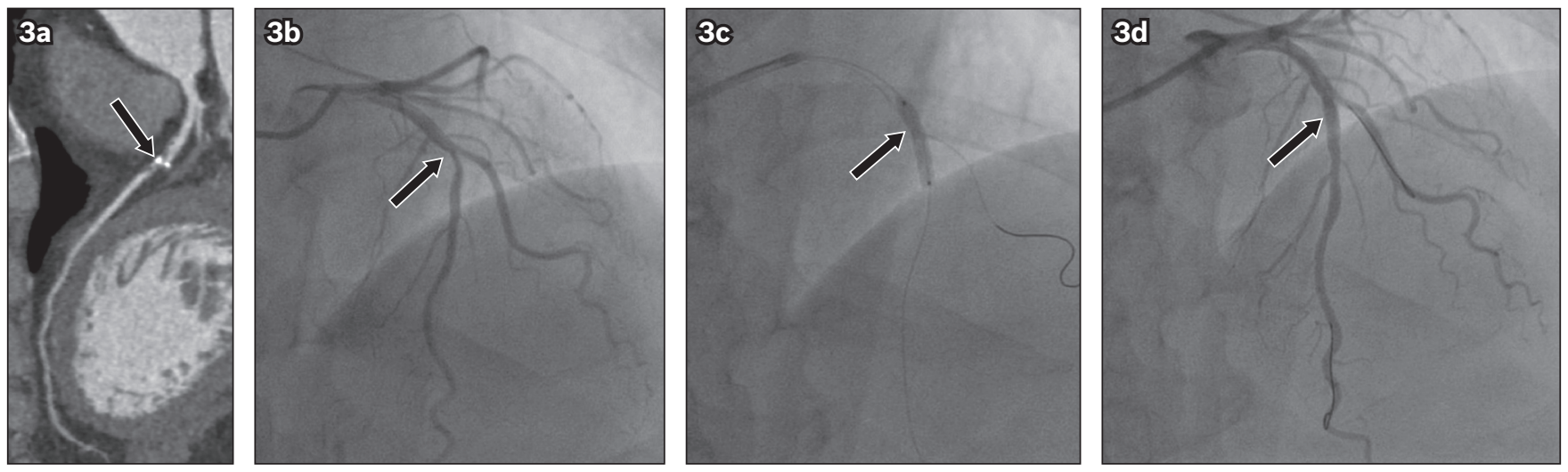

Fig. 3 A 55-year-old Chinese man presented with chest pain, without ECG changes or a rising trend of cardiac enzymes. (a) Contrast-enhanced CCTA curved planar reformation demonstrates focal severe stenosis in the mid left anterior descending artery (arrow). (b) Catheter coronary angiogram confirmed focal stenosis in the mid left anterior descending artery just distal to the origin of the circumflex artery (arrow). (c) Balloon angioplasty was performed (arrow), which achieved a (d) good result and restoration of luminal patency (arrow).

Table II. Coronary computed tomography angiography versus catheter coronary angiography accuracy studies.

\begin{tabular}{|c|c|c|c|c|c|}
\hline Study, yr & No. of patients & Sensitivity (\%) & Specificity (\%) & PPV (\%) & NPV (\%) \\
\hline Achenbach et al, $2005^{(1)}$ & 50 & 94 & 96 & 69 & 99 \\
\hline Hausleiter et al, $2007^{(29)}$ & 243 & 99 & 75 & - & 99 \\
\hline Leschka et al, $2005^{(30)}$ & 67 & 94 & 97 & 87 & 99 \\
\hline Martuscelli et al, 2004(31) & 64 & 89 & 98 & 90 & 88 \\
\hline Mollet et al, $2005^{(32)}$ & 52 & 99 & 95 & 76 & 99 \\
\hline Morgan-Hughes et al, $2005^{(33)}$ & 58 & 83 & 97 & 80 & 97 \\
\hline Pugliese et al, $2006^{(35)}$ & 35 & 99 & 96 & 78 & 99 \\
\hline Ropers et al, $2003^{(34)}$ & 77 & 92 & 93 & 79 & 97 \\
\hline Van Lingen et al, 2009(36) & 78 & 93 & 86 & 79 & 96 \\
\hline Present study, 2017 & 101 & 99 & 32 & 86 & 86 \\
\hline
\end{tabular}

NPV: negative predictive value; PPV: positive predictive value

Table III. Prognostic accuracy of coronary computed tomography angiography studies.

\begin{tabular}{|llllll|}
\hline Study, $y \mathbf{r}$ & No. of patients & Sensitivity (\%) & Specificity (\%) & PPV (\%) & NPV (\%) \\
\hline Takakuwa et al (MACE), 2011(37) & 1559 & 93.3 & 89.9 & 48.1 & 99.3 \\
\hline Nagori et al (MACE), 2015(38) & 81 & 100.0 & 95.7 & 94.7 & 100.0 \\
\hline Hoffmann et al (ACS), 2009(19) & 368 & 100.0 & 54.0 & - & 100.0 \\
\hline Present study, 2017 & 677 & 100.0 & 78.0 & 11.6 & 100.0 \\
\hline
\end{tabular}

ACS: acute coronary syndrome; MACE: major adverse cardiovascular events; NPV: negative predictive value; PPV: positive predictive value

and native coronary arteries in patients after CABG surgery. ${ }^{(24)}$ Future improvements may occur, as efforts are being made to reduce the radiation dose involved in CCTA while maintaining the required diagnostic accuracy. ${ }^{(25-27)}$

Table III shows a comparison of MACE prognostic accuracy rates in this study with those in the available published literature. The PPV of CCTA for MACE of $11.6 \%$ was extremely low, which could be due to several factors, such as aggressive medical therapy before patients developed MACE and the nature of the retrospective study versus longitudinal prospective data collection, and is reflective of the local referral pattern in which many patients with positive tests for coronary ischaemia are referred to a tertiary cardiac centre for further specialist management, including CCA.

This study had several limitations. First, prognostic information was collated retrospectively in a review of patient records rather than as a prospective, observational trial. However, the longer follow-up time in the medium term could help to compensate for this limitation. Second, not all positive CCTA patients went on to have catheter correlation, as CCA was performed only after cardiology review and was based on an assessment of the patient's clinical symptoms and other tests, including functional imaging. The study was performed with a real-world model in mind, based on an intention-todiagnose rather than an intention-to-treat model. Hence, we did not exclude cases with high coronary calcium scores (even those $>600$ units) and no studies were excluded unless the images were deemed non-diagnostic or non-interpretable. A study by Park et $\mathrm{al}^{(28)}$ suggested that although CCTA has limited value due to its low diagnostic accuracy in imaging heavily calcified plaques, it can provide information about 
mixed and non-calcified plaques that result in significant stenosis, and therefore helps in assessing the severity of CAD in patients with high calcium scores.

Third, the accuracy subgroup analysis had a small sample size, as not all cases with positive CCTA went on to have CCA. Furthermore, clinical reports for CCA rather than blinded CCA were used, which is in keeping with the normal clinical management and decision-making process of our institution. Fourth, data analysis of follow-up for MACE was limited to one institutional cluster only due to limitations in electronic health record coverage at the time of the study. If the patient did not have MACE in the same institutional cluster, it would not be identified. Hence, our results may appear to be better than in reality.

In conclusion, diagnostic CCTA at our institution had an accuracy that is in line with published trial data, as well as excellent sensitivity and NPV. The $0 \%$ event rate of MACE at a mean of one year of follow-up after negative CCTA highlights the excellent prognosis and ability to avoid further unnecessary testing. This confirms the role of CCTA as a robust, first-line, noninvasive diagnostic test for detecting CAD and providing essential information to guide management, therapy and prognostic evaluation in patients with suspected CAD. It is particularly valuable in patients with a low-to-intermediate pre-test likelihood of CAD, in whom the condition cannot be diagnosed or excluded based on clinical assessment alone.

\section{NOTE}

This abstract was published in the Singapore Medical Journal supplement, Eastern Health Alliance Scientific Meeting 2016 Transitions of Care.

\section{REFERENCES}

1. Achenbach S, Ropers D, Pohle FK, et al. Detection of coronary artery stenoses using multi-detector CT with $16 \times 0.75$ collimation and $375 \mathrm{~ms}$ rotation. Eur Heart J 2005; 26:1978-86.

2. Nielsen LH, Ortner N, Nørgaard BL, et al. The diagnostic accuracy and outcomes after coronary computed tomography angiography vs. conventional functional testing in patients with stable angina pectoris: a systematic review and metaanalysis. Eur Heart J Cardiovasc Imaging 2014; 15:961-71.

3. Pontone G, Bertella E, Mushtaq S, et al. Coronary artery disease: diagnostic accuracy of CT coronary angiography--a comparison of high and standard spatial resolution scanning. Radiology 2014; 271:688-94.

4. Atallah P, Shea R, Atallah C, et al. Diagnostic accuracy of non-invasive coronary angiography by multi-slice computed tomography. Available at: http:// www.rochestermedicalcenter.com/Original_Article_version_7.pdf. Accessed November 11, 2016.

5. Alani A, Nakanishi R, Budoff MJ. Recent improvement in coronary computed tomography angiography diagnostic accuracy. Clin Cardiol 2014; 37:428-33.

6. Sun Z, Choo GH, Ng KH. Coronary CT angiography: current status and continuing challenges. Br J Radiol 2012; 85:495-510.

7. Ferencik M, Ropers D, Abbara S, et al. Diagnostic accuracy of image postprocessing methods for the detection of coronary artery stenoses by using multidetector CT. Radiology 2007; 243:696-702.

8. Bastarrika G, Lee YS, Huda W, et al. CT of coronary artery disease. Radiology 2009; 253:317-38.

9. Gouya $\mathrm{H}$, Varenne $\mathrm{O}$, Trinquart $\mathrm{L}$, et al. Coronary artery stenosis in high-risk patients: 64-section CT and coronary angiography--prospective study and analysis of discordance. Radiology 2009; 252:377-85.

10. Catalán P, Leta R, Hidalgo A, et al. Ruling out coronary artery disease with noninvasive coronary multidetector $\mathrm{CT}$ angiography before noncoronary cardiovascular surgery. Radiology 2011; 258:426-34.

11. Scheffel H, Alkadhi H, Plass A, et al. Accuracy of dual-source CT coronary angiography: first experience in a high pre-test probability population without heart rate control. Eur Radiol 2006; 16:2739-47.
12. Meijboom WB, Mollet NR, Van Mieghem CA, et al. 64-slice CT coronary angiography in patients with non-ST elevation acute coronary syndrome. Heart 2007; 93:1386-92.

13. Nance JW Jr, Schlett CL, Schoepf UJ, et al. Incremental prognostic value of different components of coronary atherosclerotic plaque at cardiac CT angiography beyond coronary calcification in patients with acute chest pain. Radiology 2012; 264:679-90.

14. Pundziute G, Schuijf JD, Jukema JW, et al. Prognostic value of multislice computed tomography coronary angiography in patients with known or suspected coronary artery disease. J Am Coll Cardiol 2007; 49:62-70.

15. Nasis A, Meredith IT, Sud PS, et al. Long-term outcome after CT angiography in patients with possible acute coronary syndrome. Radiology 2014; 272:674-82.

16. Leschka S, Koepfli P, Husmann L, et al. Myocardial bridging: depiction rate and morphology at CT coronary angiography--comparison with conventional coronary angiography. Radiology 2008; 246:754-62.

17. Menon M, Lesser JR, Hara $\mathrm{H}$, et al. Multidetector CT coronary angiography for patient triage to invasive coronary angiography: performance and cost in ambulatory patients with equivocal or suspected inaccurate noninvasive stress tests. Catheter Cardiovasc Interv 2009; 73:497-502.

18. Metz LD, Beattie M, Hom R, et al. The prognostic value of normal exercise myocardial perfusion imaging and exercise echocardiography: a meta-analysis. J Am Coll Cardiol 2007; 49:227-37.

19. Hoffmann U, Bamberg F, Chae CU, et al. Coronary computed tomography angiography for early triage of patients with acute chest pain: the ROMICAT (Rule Out Myocardial Infarction using Computer Assisted Tomography) trial. J Am Coll Cardiol 2009; 53:1642-50.

20. Abidov A, Gallagher MJ, Chinnaiyan KM, et al. Clinical effectiveness of coronary computed tomographic angiography in the triage of patients to cardiac catheterization and revascularization after inconclusive stress testing: results of a 2-year prospective trial. J Nucl Cardiol 2009; 16:701-13.

21. Genders TS, Meijboom WB, Meijs MF, et al. CT coronary angiography in patients suspected of having coronary artery disease: decision making from various perspectives in the face of uncertainty. Radiology 2009; 253:734-44.

22. Min JK, Gilmore A, Budoff MJ, Berman DS, O'Day K. Cost-effectiveness of coronary CT angiography versus myocardial perfusion SPECT for evaluation of patients with chest pain and no known coronary artery disease. Radiology 2010; 254:801-8.

23. Suh YJ, Hong YJ, Lee HJ, et al. Accuracy of CT for selecting candidates for coronary artery bypass graft surgery: combination with the SYNTAX score. Radiology 2015; 276:390-9.

24. Chaosuwannakit N, Makarawate P, Kiatchoosakun S, Wongvipaporn C, Kuptarnond C. Diagnostic accuracy of coronary CT angiography in patients after coronary bypass surgery: evaluation of grafts and native coronary arteries. J Med Assoc Thai 2014; 97:211-9.

25. Stehli J, Fuchs TA, Bull S, et al. Accuracy of coronary CT angiography using a submillisievert fraction of radiation exposure: comparison with invasive coronary angiography. J Am Coll Cardiol 2014; 64:772-80.

26. Alkadhi H, Stolzmann P, Desbiolles L, et al. Low-dose, 128-slice, dual-source CT coronary angiography: accuracy and radiation dose of the high-pitch and the step-and-shoot mode. Heart 2010; 96:933-8.

27. Moscariello A, Takx RA, Schoepf UJ, et al. Coronary CT angiography: image quality, diagnostic accuracy, and potential for radiation dose reduction using a novel iterative image reconstruction technique-comparison with traditional filtered back projection. Eur Radiol 2011; 21:2130-8.

28. Park MJ, Jung JI, Choi YS, et al. Coronary CT angiography in patients with high calcium score: evaluation of plaque characteristics and diagnostic accuracy. Int J Cardiovasc Imaging 2011; 27 Suppl 1:43-51.

29. Hausleiter J, Meyer T, Hadamitzky M, et al. Non-invasive coronary computed tomographic angiography for patients with suspected coronary artery disease: the Coronary Angiography by Computed Tomography with the Use of a Submillimeter resolution (CACTUS) trial. Eur Heart J 2007; 28:3034-41.

30. Leschka S, Alkadhi H, Plass A, et al. Accuracy of MSCT coronary angiography with 64-slice technology: first experience. Eur Heart J 2005; 26:1482-7.

31. Martuscelli E, Romagnoli A, D'Eliseo A, et al. Accuracy of thin-slice computed tomography in the detection of coronary stenoses. Eur Heart J 2004; 25:1043-8.

32. Mollet NR, Cademartiri F, van Mieghem CA, et al. High-resolution spiral computed tomography coronary angiography in patients referred for diagnostic conventional coronary angiography. Circulation 2005; 112:2318-23.

33. Morgan-Hughes GJ, Roobottom CA, Owens PE, Marshall AJ. Highly accurate coronary angiography with submillimetre, 16 slice computed tomography. Heart 2005; 91:308-13.

34. Ropers D, Baum U, Pohle K, et al. Detection of coronary artery stenoses with thin-slice multi-detector row spiral computed tomography and multiplanar reconstruction. Circulation 2003; 107:664-6.

35. Pugliese F, Mollet NR, Runza G, et al. Diagnostic accuracy of non-invasive 64-slice CT coronary angiography in patients with stable angina pectoris. Eur Radiol 2006; 16:575-82. 
36. Van Lingen R, Kakani N, Veitch A, et al. Prognostic and accuracy data of multidetector $\mathrm{CT}$ coronary angiography in an established clinical service. Clin Radiol 2009; 64:601-7.

37. Takakuwa KM, Halpern EJ. Evaluation of a "triple rule-out" coronary CT angiography protocol: use of 64-section CT in low-to-moderate risk emergency department patients suspected of having acute coronary syndrome. Radiology 2008; 248:438-46. 38. Nagori M, Narain VS, Saran RK, Dwivedi SK, Sethi R. Efficacy of multi-detector coronary computed tomography angiography in comparison with exercise electrocardiogram in the triage of patients of low risk acute chest pain. Indian Heart J 2014; 66:435-42. 